\title{
DEVELOPMENT OF A PHOTONIC GAUGING SENSOR FOR HAZARDOUS LIQUIDS (SUMMARY)
}

\author{
Neil T. Gordon, Kaiming Zhou, David J. Webb and Alessio Cipullo ${ }^{\dagger}$ \\ Aston Institute of Photonic Technologies, Aston University, UK, ${ }^{\dagger}$ Airbus Operations Ltd, Bristol, \\ $U K$
}

\begin{abstract}
Previous papers have described the use of a Long Period Grating (LPG) as a simple photonic level sensor. We show how a useful performance of $1 \mathrm{~mm}$ resolution over $1 \mathrm{~m}$ could be obtained in a system that requires only a low-cost interrogator rather than an Optical Spectrum Analyzer.
\end{abstract}

\section{Introduction}

We are interested in developing a photonic fuel gauging sensor where the electronics can be remote from the tank and using relatively low cost, robust component. A number of techniques have been reported. In one approach, Kaliq et al. [1] described the use of a fiber, which is inscribed with a weak LPG for level sensing. The grating transfers radiation at a resonant wavelength from the core of the fiber to a cladding mode where it is lost. Thus the signal remaining in the core gradually decreases with distance. The resonant wavelength depends on the material around the fiber and hence a partially immersed LPG loses radiation at one wavelength due to the part of the LPG in air and at a second wavelength due to the submerged part. The length of the LPG needs to be greater than the required depth range and the strength of the LPG is designed to couple out most of the radiation over this range. Thus measuring the strength of the air resonance can be used to determine the length of the LPG in air and hence the depth of the liquid.

\section{Design of System}

Figure 1 shows the proposed design for the system where the superluminescent diode (SLED) is a broadband light source and the fiber interrogator is used to measure the spectrum. We have added a reflector to the end of the fiber so that the reflected radiation goes through the LPG twice and the reflectance spectrum is measured rather than the transmission. This gives a slight simplification in that only one fiber connection is needed to the tank.
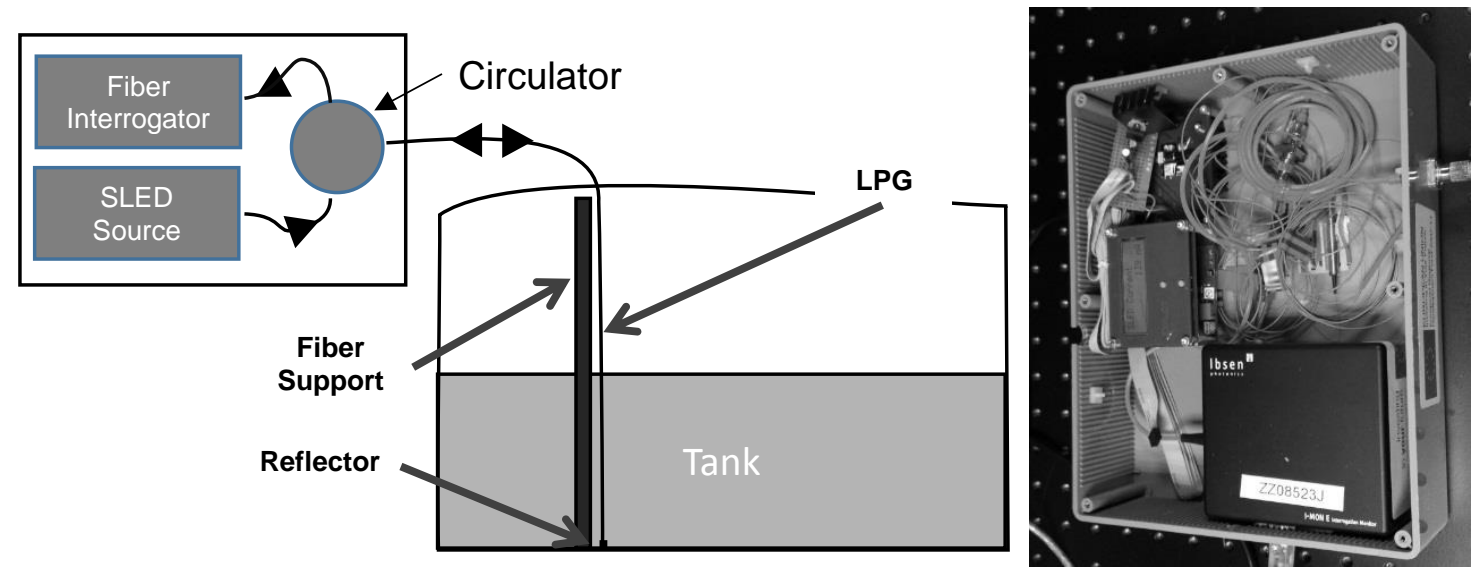

Fig 1. Design of system (left) showing the main components. The scheme requires only a single fiber input and all the electric components are remote. The electronics box is shown on the right.

\section{Modelling of System Performance}

The transmission of the LPG as a function of depth was calculated by using both the simple formula by Erdogan [2] and by using coupled mode theory and integrating across the refractive index profile [3]. The effect of the reflector was taken to be equivalent to two transmissions in series (which is different from doubling the length of the grating). The transmission of an LPG at its resonance wavelength varies with length $L$ as $T_{\text {res }}=\cos ^{2}(\kappa L)(1)$, where $k$ is the coupling strength between the core and the cladding modes. The reflected signal undergoes two transmissions and hence $T_{r e s}^{2}=\cos ^{4}(\kappa L)$. Figure 2 (left) shows this variation for 
different coupling constants. The transmission at the fuel resonance will be complimentary to the air resonance as shown in figure 2 (right).
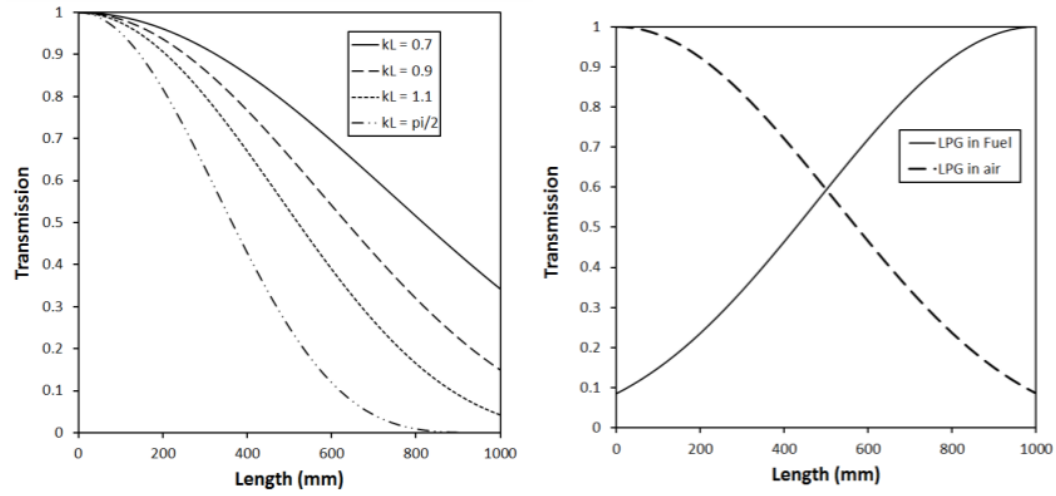

Fig 2. Transmission of a double pass through the LPG as a function of grating length (left) for different coupling strengths. The variation with length of the transmission of the air and fuel are complementary to each other as shown on the right.

The sensitivity of this technique can be estimated by differentiating equation (1) to get the change in the resonant signal per change in depth of the liquid, sens $=d T_{r e s}^{2} / d L I_{o}=-4 k I_{o} \sin (k L) \cos ^{3}(k L)$. Where $I_{o}$ is the signal measured at the resonant wavelength with no LPG. The depth resolution $\Delta L$ is the noise divided by the sensitivity $\Delta L=I_{n} /$ sens $=\left(I_{n} / I_{o}\right)\left(d T_{\text {res }}^{2} / d L\right)^{-1}=(s / n)^{-1}\left(d T_{\text {res }}^{2} / d L\right)^{-1}$. This equation can be inverted to calculate the minimum signal to noise required to achieve a given depth resolution as shown in figure 3 . By combining measurements of the transmissions at both the air and liquid resonant frequencies good performance can be achieved over the whole range. The strength of the LPG was adjusted to optimise the performance of the system. Figure 3 right gives the measured signal to noise for the system shown in figure 1 over the SLED range.
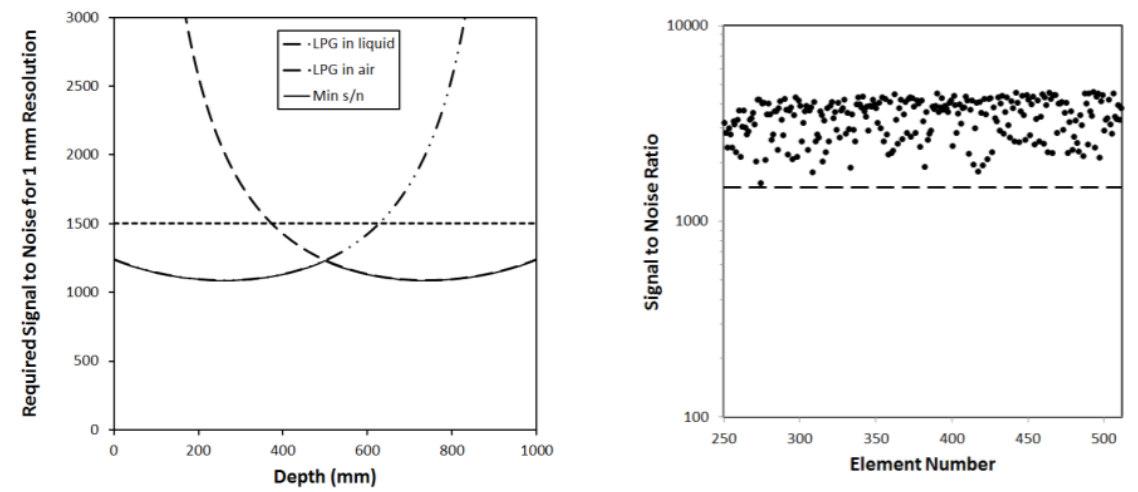

Fig 3. Signal to noise required to achieve a $1 \mathrm{~mm}$ resolution (left) and measured signal to noise for the system from figure 1 using an Ibsen IMON-E interrogator at a data rate of $16 \mathrm{~Hz}$ (right).

\section{Discussion}

The modelling and measurements above have shown that this system can give a useful performance of depth resolution and range at a good data rate using widely available and ruggedized components. Our paper will present our recent progress with this and other optical fiber based gauging techniques.

\section{References}

[1] S. Khaliq, S. W. James, and R. P. Tatam, "Fiber-optic liquid-level sensor using a long-period grating.," Opt. Lett., vol. 26, no. 16, pp. 1224-1226, 2001.

[2] T. Erdogan, “Fiber Grating Spectra," J. Light. Technol., vol. 15, no. 8, pp. 1277-1294, 1997.

[3] T. Erdogan, "Cladding-mode resonances in short- and long-period fiber grating filters," J. Opt. Soc. Am. A, vol. 14, no. 8, pp. 1760-1773, Aug. 1997. 